\title{
Vascular Structure and Inflammation in a South African Population: The SABPA Study
}

\author{
Carla Swart $^{a}$ Leandi Lammertyn ${ }^{a, b}$ Paul I. Welsh ${ }^{c}$ \\ Shani Botha-Le Roux ${ }^{a, b}$ \\ a Hypertension in Africa Research Team (HART), North-West University, Potchefstroom, \\ South Africa; ${ }^{b}$ MRC Research Unit for Hypertension and Cardiovascular Disease, \\ North-West University, Potchefstroom, South Africa; 'Institute of Cardiovascular and \\ Medical Sciences, College of Medical, Veterinary and Life Sciences, University of Glasgow, \\ Glasgow, UK
}

\section{Keywords}

C-reactive protein · Interleukin-6 · Inflammation · South Africa · Soluble urokinase plasminogen activator receptor

\begin{abstract}
Background and Objective: Inflammation plays a role in the early onset of cardiovascular disease. However, longitudinal studies on this topic, especially in South African populations, are scant. We explored whether early changes in vascular structure are associated with changes in inflammation. Methods: We investigated 303 South African teachers aged 20-65 years at two intervals, three years apart. Standardised methods were used to determine carotid intima-media thickness (IMT) and cross-sectional wall area (CSWA) as measures of vascular structure, as well as the inflammatory markers soluble urokinase plasminogen activator receptor (suPAR), C-reactive protein (CRP) and interleukin-6 (IL-6) at baseline and follow-up. Results: IMT and CSWA were higher, while CRP was lower at follow-up than at baseline. After adjusting for confounding factors, percent change in IMT was inversely associated with percent change in suPAR ( $\beta=-0.12, p=0.036$; adjusted $R^{2}=0.16$ ) only, and only in the highest tertile of percent change in suPAR $(r=-0.31 ; p=0.002)$. Conclusion: The early structural changes observed are not related to higher inflammatory levels in this South African population. Future studies are needed to investigate the possible protective effect of suPAR on early changes in vascular structure, especially with the focus on cardiovascular disease prevention.




\section{Introduction}

Cardiovascular disease (CVD) is the leading cause of disease-related mortality worldwide [1]. In addition to conventional and behavioural risk factors [2, 3], inflammation also plays a role in the enhancement of CVD development $[4,5]$. During non-pathological conditions, a balance between anti- and proinflammatory molecules exists [6]. However, low-grade inflammation, as characterised by increasing levels of proinflammatory markers such as C-reactive protein (CRP) [7], interleukin-6 (IL-6) [8] and soluble urokinase plasminogen activator receptor (suPAR) [7], results in the development of endothelial dysfunction [9]. Such events may in turn contribute to an inflammatory cascade, evident in a further increase in inflammation [10]. In time, changes in the vasculature occur, as measured by an increase in carotid intima-media thickness (IMT) and cross-sectional wall area (CSWA), thereby leading to the development of atherosclerosis and other CVDs [11-14]. This ultimately increases the risk of cardiovascular-related events and mortality $[15,16]$.

Cross-sectional studies have indicated that a positive association exists between vascular structural changes and several inflammatory markers within subjects with progressed disease, including uremic patients and subjects with a history of coronary artery disease [17, 18]. Prior studies, conducted within a South African population, also reported adverse changes in IMT and CSWA [19], as well as inflammation, such as suPAR [20] and tumour necrosis factor- $\alpha$ [19]. Nevertheless, longitudinal studies investigating the relationship between inflammation and early vascular structural changes, within a South African context, remain scant. To address this lack of information, we explored whether changes in vascular structure are associated with changes in inflammation in a South African population.

\section{Subjects and Methods}

\section{Study Design and Participants}

This study forms part of the Sympathetic Activity and Ambulatory Blood Pressure in Africans (SABPA) study. A detailed protocol has been published elsewhere [21]. We examined 303 participants at baseline (in 2008-2009) and follow-up (in 2011-2012). Teachers from the North West Province, South Africa, aged between 20 and 65 years were included. Excluded were individuals with a tympanic temperature above $37.5^{\circ} \mathrm{C}$, blood donators, those who had received vaccinations $<3$ months prior to data collection, pregnant and lactating women, subjects dependent on psychotropic substances, and HIV-infected individuals. We additionally excluded those that were lost to follow-up $(n=46)$ and those who had missing data at either baseline or follow-up $(n=35)$.

Questionnaire and Anthropometric and Physical Activity Measurements

A questionnaire was completed to obtain demographic (age, sex, race and medication use) and lifestyle (alcohol use and smoking habits) data. Waist circumference was measured in triplicate according to prescribed standardised procedures [22], and the median was reported. Obesity classification, based on waist circumference, was done according to the World Health Organisation guidelines as a waist circumference of $>102$ and $>88 \mathrm{~cm}$ for men and women, respectively [23]. Physical activity data were obtained by use of an Actical (Mini Mitter Co., Inc., Bend, ON, Canada) device at baseline and an Actiheart (CamNtech Ltd, Cambridge, UK) device at follow-up.

\section{Cardiovascular Measurements}

We determined carotid IMT, lumen diameter and CSWA as markers of vascular structure. Images of the left and right common carotid arteries were taken with the SonoSite MicroMaxx ${ }^{\circledR}$ ultrasound system (SonoSite Inc., Bothell, WA, USA) and digitally analysed with Artery Measurement Systems automated software II v1.139 (Gothenburg, Sweden) to quantify IMT and lumen diameter. The mean IMTs of the far and near walls on both sides were reported. CSWA was calculated as $\operatorname{CSWA}=\pi(d / 2+I M T f)^{2}-\pi(d / 2)^{2}$, with $d$ as the lumen diameter. 
A validated ambulatory blood pressure measurement device (CardioTens, CE0120; Meditech, Budapest, Hungary) was used to obtain systolic, diastolic, pulse and mean arterial blood pressure. Blood pressure was taken at 30-min intervals during the day (6 a.m.-10 p.m.) and 60-min intervals at night (10 p.m. -6 a.m.). Successful inflation rates of $70 \%$ or more were required for a measurement to be regarded as adequate.

Biochemical Analyses

Samples were obtained from the fasting participants and stored at $-80{ }^{\circ} \mathrm{C}$ until analyses could be performed. Plasma suPAR levels were measured with the suPARnostic ${ }^{\circledR}$ (ViroGates, Copenhagen, Denmark) test. Inter- and intra-assays were $<10.9 \%$. Serum levels of IL- 6 were measured with a Quantikine high-sensitivity enzyme-linked immunosorbent assay (R\&D Systems, Minneapolis, MN, USA). For IL-6 the intra-assay variability was $5.9 \%$ and the interassay variability was $18.9 \%$. The UniCel DxC 800 (Beckman Coulter GmbH, Krefeld, Germany) apparatus was used to determine high-sensitivity CRP, serum high-density lipoprotein cholesterol, triglycerides, sodium fluoride glucose and $\gamma$-glutamyltransferase at baseline, while the COBAS INTEGRA 400 (Roche, Switzerland) apparatus was used to analyse these markers at follow-up. CRP was analysed with the particle-enhanced turbidimetric assay method, $\gamma$-glutamyltransferase with the enzyme rate method, and high-density lipoprotein cholesterol and triglycerides with the homogeneous enzymatic colorimetric assay method.

\section{Statistical Analyses}

TIBICO ${ }^{\circledR}$ Statistica ${ }^{\mathrm{TM}}$ version 13.3 (TIBICO Software Inc., Palo Alto, CA, USA) was used to perform statistical analyses. Normally distributed, continuous data are presented as arithmetic means \pm standard deviation. Where non-gaussian data are concerned, data were log-transformed and are presented as geometric means with 5 th and 95th percentiles. Categorical data are presented as proportions. We additionally adjusted for mean arterial pressure with regard to IMT, CSWA and lumen diameter. Possible interactions of sex and race with the association of vascular structure with inflammatory markers were tested by means of multiple regression analyses. Differences in means between baseline and follow-up were determined with dependent $t$ tests for continuous, and with McNemar tests for categorical data.

Relationships were established with Pearson correlations, and multiple linear regression analyses were performed to determine independent relationships. In all cases, percent change in IMT was regarded as the main dependent, and either baseline or percent change in inflammatory markers as the main independent variable, to account for the regression to the mean. Pearson correlations were adjusted for sex, age and waist circumference and were performed on the total group, as well as on the groups stratified by tertiles of percent change in suPAR and CRP, respectively. Covariates used for the multiple regression models included race, sex, and age, as well as baseline waist circumference, $\gamma$-glutamyltransferase, glucose, triglyceride-to-high-density lipoprotein ratio, mean arterial pressure, and self-reported tobacco and antiinflammatory medication use. All measurements were two-tailed, and $p \leq 0.05$ was used to indicate statistical significance.

\section{Results}

The baseline and follow-up characteristics are described in Table 1. Of the 303 South Africans included, 49.5\% were men and 39.9\% were Black. Further, anthropometric measures, pulse pressure, triglyceride-to-high-density lipoprotein cholesterol ratio, total energy expenditure, and antihypertensive and statin medication use were all higher (all $p \leq$ $0.015)$, while diastolic blood pressure, glucose, $\gamma$-glutamyltransferase and tobacco use were lower (all $p \leq 0.001$ ), at follow-up than at baseline. With regard to vascular structure (IMT, CSWA and lumen diameter), all markers were higher at follow-up than at baseline (all $p<$ 0.001). Regarding inflammatory markers, CRP was lower at follow-up than at baseline $(p<$ $0.001)$.

In the multiple regression analyses (Table 2), an inverse association was observed between percent change in IMT and change in suPAR such that a $1 \%$ increase in suPAR was associated with a $0.12 \%$ decrease in IMT $\left(\beta=-0.12, p=0.036\right.$; adjusted $\left.R^{2}=0.16\right)$, while no 
Table 1. Characteristics of the study population $(n=303)$

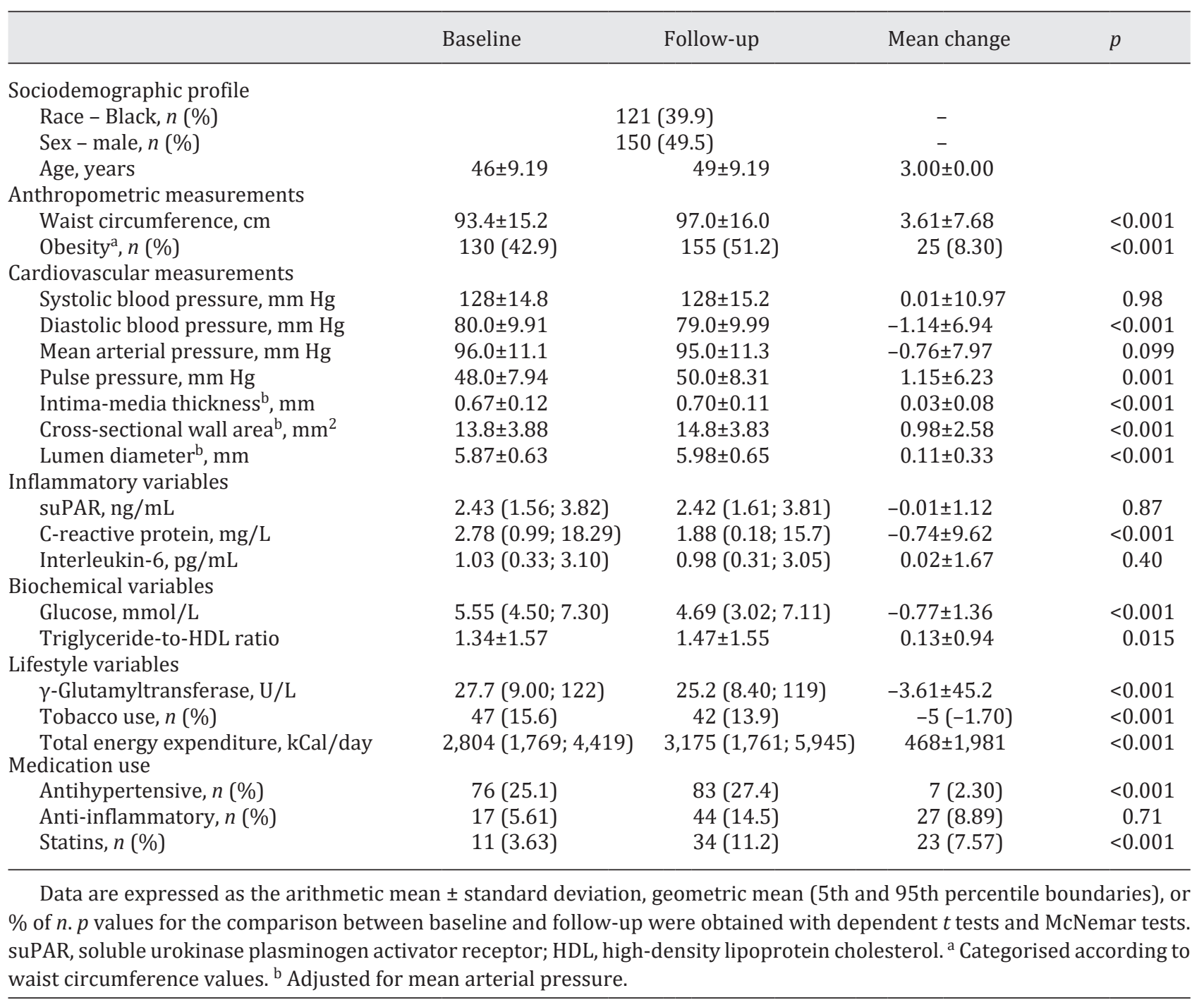

associations were obtained between percent change in IMT and baseline suPAR. Percent change in CSWA was associated with baseline CRP $(\beta=0.75, p=0.042)$, but not with percent change in CRP $(\beta=0.003, p=0.98)$.

Further, in the partial correlation analyses (Fig. 1; Table 3), we found an inverse association between percent change in IMT and percent change in suPAR within the total group $(r=-0.13 ; p=0.025)$ and in the "highest percent change in suPAR tertile" $(r=-0.31 ; p=0.002)$. No associations were found between percent change in IMT and percent change in CRP.

We additionally tested for the effect of sex and race, respectively, on the association between percent change in IMT and percent change in inflammatory markers, but we found no significant effect (Table 4).

We also performed sensitivity analyses to test for the effect of statin and antihypertensive medication use, respectively, on the association between IMT and inflammatory markers, but we found no significant effect (results not shown). 
Table 2. Independent associations of percent change in IMT and CSWA, respectively, with baseline and percent change in inflammatory markers, respectively, in the total group $(n=292)$

\begin{tabular}{llll}
\hline & Adjusted $R^{2}$ & $\beta(95 \% \mathrm{CI})$ & $p$ \\
\hline $\begin{array}{l}\text { Percent change in IMT } \\
\text { Baseline }\end{array}$ & & \\
$\quad$ suPAR, ng/mL & 0.16 & $1.09(-1.15 ; 3.32)$ & 0.53 \\
$\quad$ C-reactive protein, mg/L & 0.17 & $0.76(-1.49 ; 3.01)$ & 0.051 \\
$\quad$ Interleukin-6, pg/mL & 0.16 & $0.90(-1.34 ; 3.13)$ & 0.40 \\
Percent change & & \\
$\quad$ suPAR, ng/mL & 0.16 & $-0.12(-0.22 ;-0.01)$ & 0.036 \\
$\quad$ C-reactive protein, mg/L & 0.15 & $-0.01(-0.12 ; 0.10)$ & 0.89 \\
$\quad$ Interleukin-6, pg/mL & 0.15 & $-0.04(-0.15 ; 0.07)$ & 0.52 \\
\hline Percent change in CSWA & & & \\
Baseline & & $0.94(-1.29 ; 3.18)$ & 0.67 \\
$\quad$ suPAR, ng/mL & 0.11 & $0.75(-1.51 ; 3.01)$ & 0.042 \\
$\quad$ C-reactive protein, mg/L & 0.13 & $1.35(-0.89 ; 3.59)$ & 0.42 \\
$\quad$ Interleukin-6, pg/mL & 0.11 & $-0.03(-0.09 ; 0.03)$ & 0.35 \\
Percent change & & $0.003(-0.02 ; 0.02)$ & 0.98 \\
$\quad$ suPAR, ng/mL & 0.11 & $-0.09(-0.22 ; 0.05)$ & 0.78 \\
$\quad$ C-reactive protein, mg/L & 0.11 & 0.11 & \\
Interleukin-6, pg/mL & & & \\
\hline
\end{tabular}

The models include age, race, sex, waist circumference, glucose, high-density lipoprotein cholesterol, total energy expenditure, $\gamma$-glutamyltransferase, tobacco use, anti-inflammatory medication use and mean arterial pressure. $\beta$, partial regression coefficient; $95 \%$ CI, $95 \%$ confidence interval of $\beta$; IMT, intima-media thickness; suPAR, soluble urokinase plasminogen activator receptor; CSWA, cross-sectional wall area.

Table 3. Relationship of percent change in IMT to percent change in suPAR and C-reactive protein, respectively, stratified by tertiles of suPAR and CRP

\begin{tabular}{|c|c|c|c|c|c|c|c|c|}
\hline & \multicolumn{2}{|c|}{ Total group } & \multicolumn{2}{|c|}{ 1st tertile } & \multicolumn{2}{|c|}{ 2nd tertile } & \multicolumn{2}{|c|}{ 3rd tertile } \\
\hline & $r$ & $p$ & $r$ & $p$ & $r$ & $p$ & $r$ & $p$ \\
\hline \multicolumn{9}{|l|}{ Change in IMT } \\
\hline suPAR & -0.13 & 0.025 & -0.18 & 0.10 & 0.07 & 0.48 & -0.31 & 0.002 \\
\hline C-reactive protein & -0.07 & 0.26 & -0.13 & 0.22 & 0.10 & 0.33 & -0.14 & 0.19 \\
\hline
\end{tabular}

$p$ values were obtained with partial correlations. Adjusted for sex, age and waist circumference. IMT, intima-media thickness; suPAR, soluble urokinase plasminogen activator receptor.

\section{Discussion}

We investigated whether changes in vascular structure are associated with changes in inflammation over a 3-year period. We found that a decrease in IMT was associated with an increase in suPAR, specifically in the highest tertile of percent change in suPAR. Despite positive associations of CRP with an adverse vascular phenotype at baseline, change in CRP was not associated with change in vascular phenotype.

A major advantage of our study is its ability to contrast cross-sectional with prospective data. As such, a trajectory for a causal exposure might provide a stronger signal than an 


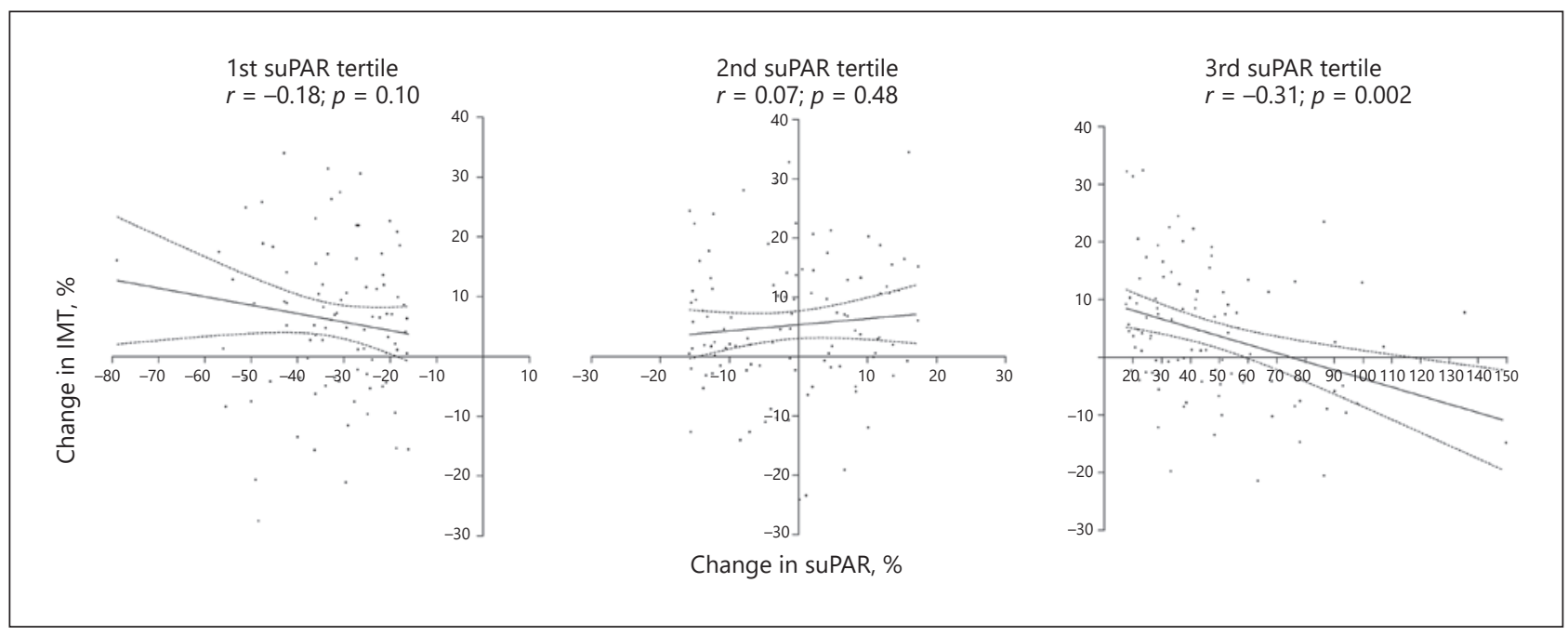

Fig. 1. Relationship of $\%$ change in IMT with $\%$ change in suPAR, stratified by tertiles of suPAR. $p$ values were obtained with partial correlations. Adjusted for sex, age and waist circumference. IMT, intima-media thickness; suPAR, soluble urokinase plasminogen activator receptor.

Table 4. Effect of race and sex on the association between percent change in IMT and baseline inflammatory markers in the total group

\begin{tabular}{llll}
\hline & Adjusted $R^{2}$ & $\beta(95 \% \mathrm{CI})$ & $p$ \\
\hline $\begin{array}{l}\text { Interaction with race } \\
\quad\end{array}$ & & & \\
$\quad$ suPAR, ng/mL & 0.15 & $1.46(-1.46 ; 4.38)$ & 0.34 \\
$\quad$ C-reactive protein, mg/L & 0.15 & $1.04(-1.31 ; 3.40)$ & 0.82 \\
$\quad$ Interleukin-6, pg/mL & 0.14 & $1.03(-1.35 ; 3.41)$ & 0.89 \\
Interaction with sex & & & \\
$\quad$ suPAR, ng/mL & 0.01 & $2.46(-0.64 ; 5.56)$ & 0.050 \\
$\quad$ C-reactive protein, mg/L & 0.05 & $1.04(-1.49 ; 3.56)$ & 0.88 \\
$\quad$ Interleukin-6, pg/mL & 0.01 & $1.22(-1.15 ; 3.58)$ & 0.30 \\
\hline
\end{tabular}

$\beta$, partial regression coefficient; $95 \% \mathrm{CI}$, 95\% confidence interval of $\beta$; IMT, intima-media thickness; suPAR, soluble urokinase plasminogen activator receptor.

exposure at a fixed time point. Even though percentage change in IMT, but not CSWA, was associated with percentage change in suPAR, this association was inverse, and neither IMT nor CSWA was associated with either CRP or IL-6. This result was unexpected, since a 15-year study including men and women aged 45-68 years found that suPAR was associated with an increase in carotid plaque and a higher prevalence of coronary artery disease [24]. This positive association with suPAR was further confirmed in other cross-sectional studies [18, 25]. However, it should be considered that these studies were conducted on subjects with progressed CVD [23] or related diseases [18, 25], which is not the case in our population sample. The participants in our study had relatively normal IMT values, seeing that only $4 \%$ had follow-up IMT values of $>0.90 \mathrm{~mm}$, which is the proposed cut-off point for subclinical IMT, as prescribed by the 2016 European guidelines on CVD prevention in clinical practice [26]. 
Nonetheless, we expect that a positive association between vascular structural changes and suPAR may only become evident in the longer term, once the vascular structure has progressed to such a stage that atherosclerosis and other associated CVDs have become evident. Our novel results, showing an inverse relationship between IMT and suPAR, especially when suPAR levels increase more, underline the necessity for more research to determine the possible mechanism behind this relationship, especially at early vascular disease stages. Other factors may also influence this relationship. For instance, both antiinflammatory and antioxidant biomarkers [27] have been found to play a protective role during the early stages of CVDs, but the literature investigating their effects on suPAR, and specifically their effect on the association between early changes in vascular structure and proinflammatory markers, is scarce.

The absence of associations of IMT with CRP and IL-6, respectively, in our study supports previous findings. In another 3-year study, which included 3,122 subjects from Europe, no association was found between early progression of IMT and baseline CRP after adjusting for conventional risk factors [28]. However, to the best of our knowledge, previous studies have not yet established a relationship between percent change in CSWA and baseline CRP. Furthermore, a study conducted in the USA found that the relationship between baseline IMT and CRP weakened after adjusting for risk factors such as smoking, blood pressure and diabetes mellitus [29]. With regard to IL-6, a stimulator of CRP production [30], the Rotterdam study with a sample size of 7,983 subjects aged 55 years and older also found no independent association between IL-6 and IMT [31]. Similar results were obtained in an Australian study, where IL- 6 was only found to be a predictor of IMT if CRP, fibrinogen and monocyte count were included in the linear regression model. However, after adjusting for conventional risk factors, the association between IMT and IL-6 lost significance [32]. These findings may indicate that neither CRP nor IL-6 have an independent effect on an increase in IMT but may rather facilitate the effect that some conventional risk factors such as obesity and tobacco and alcohol use have on vascular structure.

Notably, it is known that suPAR and CRP may represent different processes in vascular inflammation [13]. suPAR, which is secreted by smooth muscle cells and endothelial cells [33], plays a role as marker of inflammation in the vascular wall [13] and is regarded as a better marker of CVD [20] and cardiovasculature-related mortality [34] than CRP. CRP, on the other hand, originates from adipose tissue, is synthesised by the liver and is therefore rather associated with adiposity-related inflammation [13].

The strength of this study lies within its longitudinal nature and the inclusion of data which are limited within a South African research setting. Even though this study may be limited by the small sample size, power analyses proved this sample size to be sufficient. Our study design further contributed to this being a well-controlled group. Limitations of the current analysis include the observational nature of the data; despite the prospective design, causal inference is challenging. Change measures are challenging to analyse as additive errors from baseline and follow-up contribute to misclassification of exposure changes. It is possible that undetermined confounders, such as the specific dosage of drugs used by each participant and the intensity of their lifestyle modifications, may have accounted for the observed associations or masked real underlying associations. However, we did account for a variety of other potential confounders in our analyses.

In conclusion, we found that thickening of the vascular wall was related to a decrease in the inflammatory marker suPAR. More in-depth research is recommended to investigate the specific, possible protective role of suPAR in early changes in vascular structure within the South African population. 


\section{Acknowledgements}

The SABPA study would not have been possible without the voluntary collaboration of the participants, the Department of Education, North West Province, South Africa, or the valuable contributions from co-investigators and technical staff. We also acknowledge Jesper Eugen-Olsen (Copenhagen University Hospital, Hvidovre, Denmark), who is the founder, shareholder and board member of ViroGates A/S, Denmark, the company that produces the suPARnostic ${ }^{\circledR}$ assay, for performing the suPAR analyses used in this study. Any opinion, findings and conclusions or recommendations expressed in this material are those of the authors; therefore, the funders do not accept any liability regarding this study.

\section{Statement of Ethics}

Written informed consent was obtained for each participant prior to baseline and follow-up data collections. This study was approved by the Ethics Committee of the North-West University, Potchefstroom (NWU00051-17-A1) and complies with the Declaration of Helsinki.

\section{Disclosure Statement}

The authors have no conflicts of interest to declare.

\section{Funding Sources}

The SABPA study was financially supported by the Metabolic Syndrome Institute in France, the NorthWest University, South African Medical Research Council, National Research Foundation, PA \& Alize Malan Trust, North-West Department of Education, South Africa, and Roche Diagnostics, South Africa.

\section{Author Contributions}

Conceptualisation: S. Botha-Le Roux and C. Swart; methodology: all authors; statistical analysis: C. Swart; writing of the article: all authors.

\section{References}

1 World Health Organization. Cardiovascular diseases (CVDs) fact sheet 2017 [updated May 2017]. Available from: http://www.who.int/mediacentre/factsheets/fs317/en/ [accessed 2017 Aug 15].

2 Hamer M, Malan L, Schutte AE, Huisman HW, van Rooyen JM, Schutte R, et al. Conventional and behavioral risk factors explain differences in sub-clinical vascular disease between black and Caucasian South Africans: the SABPA study. Atherosclerosis. 2011 Mar;215(1):237-42.

3 van Rooyen JM, Kruger HS, Huisman HW, Wissing MP, Margetts BM, Venter CS, et al. An epidemiological study of hypertension and its determinants in a population in transition: the THUSA study. J Hum Hypertens. 2000 Dec;14(12):779-87.

4 Norman R, Bradshaw D, Schneider M, Pieterse D, Groenewald P. Revised burden of disease estimates for the comparative risk factor assessment, South Africa 2000. Cape Town: Medical Research Council; 2006.

5 Lorenz MW, Markus HS, Bots ML, Rosvall M, Sitzer M. Prediction of clinical cardiovascular events with carotid intima-media thickness: a systematic review and meta-analysis. Circulation. 2007 Jan;115(4):459-67.

6 Sprague AH, Khalil RA. Inflammatory cytokines in vascular dysfunction and vascular disease. Biochem Pharmacol. 2009 Sep; 78(6):539-52.

7 Thunø M, Macho B, Eugen-Olsen J. suPAR: the molecular crystal ball. Dis Markers. 2009;27(3):157-72.

8 Tedgui A, Mallat Z. Cytokines in atherosclerosis: pathogenic and regulatory pathways. Physiol Rev. 2006 Apr; 86(2):515-81.

9 Zhang C. The role of inflammatory cytokines in endothelial dysfunction. Basic Res Cardiol. 2008 Sep;103(5): 398-406.

10 Mackay CR. Chemokines: immunology's high impact factors. Nat Immunol. 2001 Feb;2(2):95-101. 
11 Cao JJ, Thach C, Manolio TA, Psaty BM, Kuller LH, Chaves PH, et al. C-reactive protein, carotid intima-media thickness, and incidence of ischemic stroke in the elderly: the Cardiovascular Health Study. Circulation. 2003 Jul;108(2):166-70.

12 Larsson PT, Hallerstam S, Rosfors S, Wallén NH. Circulating markers of inflammation are related to carotid artery atherosclerosis. Int Angiol. 2005 Mar;24(1):43-51.

13 Lyngbæk S, Sehestedt T, Marott JL, Hansen TW, Olsen MH, Andersen O, et al. CRP and suPAR are differently related to anthropometry and subclinical organ damage. Int J Cardiol. 2013 Aug;167(3):781-5.

14 Ross R. Atherosclerosis - an inflammatory disease. N Engl J Med. 1999 Jan;340(2):115-26.

15 Bots ML, Hoes AW, Koudstaal PJ, Hofman A, Grobbee DE. Common carotid intima-media thickness and risk of stroke and myocardial infarction: the Rotterdam Study. Circulation. 1997 Sep;96(5):1432-7.

16 Sanchis-Gomar F, Perez-Quilis C, Leischik R, Lucia A. Epidemiology of coronary heart disease and acute coronary syndrome. Ann Transl Med. 2016 Jul;4(13):256.

17 Rueda-Clausen CF, López-Jaramillo P, Luengas C, del Pilar Oubiña M, Cachofeiro V, Lahera V. Inflammation but not endothelial dysfunction is associated with the severity of coronary artery disease in dyslipidemic subjects. Mediators Inflamm. 2009;2009:469169.

18 Pawlak K, Mysliwiec M, Pawlak D. The urokinase-type plasminogen activator/its soluble receptor system is independently related to carotid atherosclerosis and associated with CC-chemokines in uraemic patients. Thromb Res. 2008;122(3):328-35.

19 Hamer M, von Känel R, Reimann M, Malan NT, Schutte AE, Huisman HW, et al. Progression of cardiovascular risk factors in black Africans: 3 year follow up of the SABPA cohort study. Atherosclerosis. 2015 Jan;238(1): 52-4.

20 Botha S, Fourie CM, Schutte R, Eugen-Olsen J, Schutte AE. Soluble urokinase plasminogen activator receptor and hypertension among black South Africans after 5 years. Hypertens Res. 2015 Jun;38(6):439-44.

21 Malan L, Hamer M, Frasure-Smith N, Steyn HS, Malan NT. Cohort profile: Sympathetic Activity and Ambulatory Blood Pressure in Africans (SABPA) prospective cohort study. Int J Epidemiol. 2015 Dec;44(6):1814-22.

22 Marfell-Jones MJ, Stewart AD, de Ridder JH. International standards for anthropometric assessment. Wellington, New Zealand: International Society for the Advancement of Kinanthropometry; 2012.

23 World Health Organization. Waist circumference and waist-hip ratio: report of a WHO expert consultation, Geneva, 8-11 December 2008. Geneva: World Health Organization; 2011.

24 Persson M, Östling G, Smith G, Hamrefors V, Melander O, Hedblad B, et al. Soluble urokinase plasminogen activator receptor: a risk factor for carotid plaque, stroke, and coronary artery disease. Stroke. 2014 Jan;45(1): 18-23.

25 Pawlak K, Pawlak D, Mysliwiec M. Tissue factor and urokinase-type plasminogen activator system are related to the presence of cardiovascular disease in hemodialysis patients. Thromb Res. 2007;120(6):871-6.

26 Piepoli MF, Hoes AW, Agewall S, Albus C, Brotons C, Catapano AL, et al.; ESC Scientific Document Group. 2016 European Guidelines on cardiovascular disease prevention in clinical practice: The Sixth Joint Task Force of the European Society of Cardiology and Other Societies on Cardiovascular Disease Prevention in Clinical Practice (constituted by representatives of 10 societies and by invited experts). Developed with the special contribution of the European Association for Cardiovascular Prevention \& Rehabilitation (EACPR). Eur Heart J. 2016 Aug;37(29):2315-81.

27 van Rooyen J, Schoeman Z, Mels C. The difference in glutathione peroxidase activity on arteries of a bi-ethnic population: the SABPA study. Artery Res. 2016;16:63.

28 Lorenz MW, Karbstein P, Markus HS, Sitzer M. High-sensitivity C-reactive protein is not associated with carotid intima-media progression: the carotid atherosclerosis progression study. Stroke. 2007 Jun;38(6):1774-9.

29 Sitzer M, Markus HS, Mendall MA, Liehr R, Knorr U, Steinmetz H. C-reactive protein and carotid intimal medial thickness in a community population. J Cardiovasc Risk. 2002 Apr;9(2):97-103.

30 Toniatti C, Arcone R, Majello B, Ganter U, Arpaia G, Ciliberto G. Regulation of the human C-reactive protein gene, a major marker of inflammation and cancer. Mol Biol Med. 1990 Jun;7(3):199-212.

31 van der Meer IM, de Maat MP, Bots ML, Breteler MM, Meijer J, Kiliaan AJ, et al. Inflammatory mediators and cell adhesion molecules as indicators of severity of atherosclerosis: the Rotterdam Study. Arterioscler Thromb Vasc Biol. 2002 May;22(5):838-42.

32 Chapman CM, Beilby JP, McQuillan BM, Thompson PL, Hung J. Monocyte count, but not C-reactive protein or interleukin-6, is an independent risk marker for subclinical carotid atherosclerosis. Stroke. 2004 Jul;35(7): 1619-24.

33 Intzilakis T, Hartmann G, Mouridsen MR, Eugen-Olsen J, Kumarathurai P, Madsbad S, et al. Soluble urokinase plasminogen activator receptor, C-reactive protein and triglyceride are associated with heart rate variability in non-diabetic Danes. Eur J Clin Invest. 2013 May;43(5):457-68.

34 Botha S, Fourie CM, Schutte R, Eugen-Olsen J, Pretorius R, Schutte AE. Soluble urokinase plasminogen activator receptor as a prognostic marker of all-cause and cardiovascular mortality in a black population. Int J Cardiol. 2015 Apr;184:631-6. 